(2) Open Access Full Text Article

\title{
Improving current immunoglobulin therapy for patients with primary immunodeficiency: quality of life and views on treatment
}

This article was published in the following Dove Press journal:

Patient Preference and Adherence

2 May 2014

Number of times this article has been viewed

\author{
Teresa Espanol' \\ Johan Prevot ${ }^{2}$ \\ Jose Drabwell ${ }^{2}$ \\ Seema Sondhi ${ }^{3}$ \\ Laurence Olding ${ }^{4}$ \\ 'Immunology Unit, Vall d'Hebron \\ University Hospital, Barcelona, Spain \\ ${ }^{2}$ International Patient Organisation \\ for Primary Immunodeficiencies, \\ Cornwall, UK; ${ }^{3}$ Baxter Healthcare SA, \\ Zurich, Switzerland; ${ }^{4}$ Bryter, \\ London, UK
}

Correspondence:Teresa Espanol Immunology Unit, Vall d'Hebron University Hospital, Barcelona, Spain Tel +34680759566

Email espaol.teresa2@gmail.com
Background: Subcutaneous or intravenous immunoglobulin replacement is the mainstay of treatment for most patients with primary immunodeficiency disease (PID). The purpose of this study was to gain an understanding of how existing PID therapies affect patient lives and to identify desired improvements to immunoglobulin treatments.

Methods: An online questionnaire was made available through the International Patient Organisation for Primary Immunodeficiencies to patients with PID and their caregivers regarding current treatment satisfaction, living with PID, and patient preferences using a conjoint approach. Health-related quality of life was canvassed via questionnaires using the Short Form 12 Health Survey and EuroQoL 5 Dimensions.

Results: A total of 300 responded to the survey ( $72 \%$ patients with PID and $28 \%$ caregivers) from across 21 countries, mostly the UK, Sweden, Canada, France, Germany, and Spain. Fifty-three percent and $45 \%$ of patients received intravenous and subcutaneous therapy, respectively. Most respondents (76\%) were satisfied with their current treatment, reflecting the benefits that immunoglobulin therapy provides for patient health and well-being. However, patients remained below the physical and mental well-being norms for health-related quality of life as determined by the questionnaire. All respondents expressed a desire for 4-weekly infusions, the ability to administer these at home, self-administration, shorter duration of administration, and fewer needle sticks.

Conclusion: The results of this survey highlight the importance of providing access to different treatment options and modes of administration to ensure individual patient needs are best met.

Keywords: primary immunodeficiency, immunoglobulins, quality of life, patient needs, patient satisfaction, conjoint analysis

\section{Introduction}

Primary immunodeficiency diseases (PIDs) are a heterogeneous group of rare disorders characterized by poor or absent function in one or more components of the innate and adaptive immune systems resulting in increased susceptibility to infection. ${ }^{1} \mathrm{~W}$ ith more than 15 novel disease entities added to the updated classification in 2011, more than 165 different PIDs are recognized by the International Union of Immunological Societies Primary Immunodeficiency Diseases Classification Committee. ${ }^{1}$ Most PIDs are caused by inherited defects. ${ }^{2,3}$

Treatment of PID involves, along with other therapies, both supportive (eg, immunoglobulin replacement therapy) and definitive (eg, bone marrow/hematopoietic stem cell transplantation, gene therapy) strategies, depending on the nature of the gene defect. ${ }^{2}$ 
Immunoglobulin replacement is the mainstay of treatment for the majority of patients. ${ }^{4}$ Routinely, immunoglobulin therapy is administered intravenously (IVIg) or subcutaneously (SCIg) at regular intervals to increase serum immunoglobulin $\mathrm{G}$ trough levels to physiologic concentrations and to protect against bacterial infection. ${ }^{5}$

IVIg is usually administered once every 3-4 weeks in hospital over 2-4 hours. Consequently, administration of IVIg leads to a rapid and high rise in the serum immunoglobulin G concentration followed by a rapid fall over the next few days; mild adverse events can be associated with the very high peaks experienced after large intravenous boluses. ${ }^{5}$

SCIg regimens are typically given once or twice weekly over 1-2 hours, can be self-administered at home using a programmable pump, and are associated with a reduced risk of systemic adverse events compared with IVIg., ${ }^{6,7}$ The smaller doses given at more frequent intervals with SCIg result in more stable and higher serum immunoglobulin G trough concentrations than with IVIg. ${ }^{5}$ The primary limitation of SCIg is the inability of tissues to accept large volumes of fluid rapidly; ${ }^{5}$ therefore, patients require frequent administration of a smaller volume at multiple sites. Frequency of injections and local injection site reactions are the primary reasons for patients reporting less satisfaction with subcutaneous therapy. ${ }^{8}$

Studies investigating health-related quality of life (HRQoL) issues in patients with PID suggest that immunoglobulin replacement therapy administered by the intravenous or subcutaneous route improves HRQoL, and that many of the improvements relate to reduced infection rates and less fear of future infections. ${ }^{9}$ However, the aforementioned limitations of current IVIg and SCIg treatments indicate that new treatments would have the potential for further improvement.

The objective of this multinational study was to gain a deeper understanding of: current immunoglobulin replacement therapy and how it affects patients' lives; the impact of PID on HRQoL in patients on immunoglobulin therapy; and improvement in treatment desired by patients on immunoglobulin therapy, including patient preferences evaluated by conjoint analysis.

\section{Materials and methods}

Participants in this study were patients with PID on regular treatment or their caregivers who participated on the patient's behalf (no age limits were applied). Patients/caregivers were invited to complete the survey through National Member Organisations (NMOs) of the International Patient Organisation for Primary Immunodeficiencies (IPOPI). Recruitment was done using electronic and postal methods, including mailing lists, newsletters, and promotion on NMO websites. Participation was voluntary and no compensation was provided; respondents provided implied consent by their participation in the survey.

The research was conducted from April 8, 2011 to October 17, 2011 by an independent market research agency (Bryter, London, UK) in accordance with privacy and data protection codes of conduct. The survey comprised a 30-minute online questionnaire, which was available in six languages (English, French, German, Italian, Spanish, and Portuguese). The survey utilized different elicitation techniques, including grid questions, coded lists, and rating scales with questions appropriately phrased for patients and caregivers. Patient and caregiver versions were reviewed and approved by the IPOPI executive board to ensure that they were appropriate for the audiences interviewed.

The questionnaire sought to establish a patient's current treatment, including route of administration, the key personnel involved in making treatment decisions, dose frequency, and site of care. It also explored patient satisfaction regarding treatment, treatment-related adverse events, and impact of PID and treatment on HRQoL. Patient preferences were determined using a conjoint approach. Five attributes common to immunoglobulin treatment and of importance to patients and caregivers were assessed: mode of administration (self-administration or administration by a health care professional), frequency of administration (one, two, or four times a month), location (home versus surgery, doctor's office, clinic, or hospital), number of needle sticks per treatment (one, two, or four), and treatment duration (2, 4, or 6 hours). Selection of attributes was defined in a previously conducted US-based study. ${ }^{10}$

The survey also incorporated validated HRQoL scales through the 12-Item Short Form Health Survey (SF-12v2; adults aged $\geq 19$ years) and 10-Item Short Form Health Survey (SF-10v2; caregivers for children aged $<19$ years). ${ }^{11,12}$ The SF-12v2 is a shorter version of the SF-36v2 Health Survey and measures functional health and well-being from the patient's point of view by just 12 questions, covering the same eight health domains as the SF-36v2. The SF-10v2 is a parent-completed survey containing ten questions adapted from the Child Health Questionnaire. Using norm-based scoring, each health domain scale and summary of physical and psychosocial health measures, from 0 (worse health) to 100 (better health), were scored to have the same mean (50) and standard deviation (10) as in the general US population. Patient health profiles were evaluated using 
the EuroQoL 5 Dimensions (EuroQol Group, Rotterdam, the Netherlands), a self-assessment questionnaire that produces a self-reported description of the patient's health in five dimensions, ie, mobility, self-care, usual activities, pain/discomfort, and anxiety/depression. ${ }^{13}$ The patients were asked to rate their current level of function (severe, moderate, or none) in each dimension and indicate their health state on a visual analog scale from 0 (worst imaginable health state) to 100 (best imaginable health state). The surveys were conducted online. All questions had to be answered before the survey could be considered completed, eliminating the possibility of missing values.

Data were collected online and, with the exception of data for the conjoint analysis, were subjected to univariate analyses. Group comparisons were analyzed at the overall (all respondent) level and compared and contrasted by pertinent subgroups, ie, those receiving SCIg versus IVIg, caregiver versus patient respondents, and by geographic location (region and/or country). These results were tested for statistical significance using an unpaired $t$-test at the 95\% confidence level.

Preferences for treatment attributes were analyzed using a conjoint analysis approach. Conjoint analysis comprises a category of preference research techniques for eliciting and quantifying preferences, and allows for trade-offs to be taken into consideration when quantifying the relative importance that patients or caregivers assign to treatment attributes or treatment outcomes. The responses to the choice questions were analyzed using random-parameters logit models, which account for random variations in preferences for each sample (patients and caregivers); ${ }^{14}$ the choice models were estimated using Sawtooth Software (Sawtooth Software Inc, Orem, UT, USA), which is a specialist software program for choicebased conjoint analysis. The resulting parameter estimates quantified the relative preference weight of each attribute level within $95 \%$ confidence intervals. When confidence intervals did not overlap for adjacent levels in a particular attribute, the mean estimates were significantly different from each other at the $5 \%$ level $(P<0.05)$. To facilitate the interpretation of the results and compare the two samples, preference weights were scaled for each sample between 0 and 10 , where the highest parameter was assigned a value of 10 and the lowest 0 . All other parameters were scaled between these two parameters. The vertical distance between the best and worst level (ie, the difference in model coefficients) of each attribute provided a measure of the overall mean relative importance of that attribute to respondents (across the ranges presented in the survey). ${ }^{15-17}$

\section{Results \\ Respondent demographics}

A total of 300 respondents (216 patients and 84 caregivers) completed the survey. Respondents were from the UK $(n=59)$, Sweden $(n=34)$, Canada $(n=31)$, France $(n=31)$, Germany $(n=31)$, Spain $(n=22)$, Portugal $(n=21)$, Argentina $(n=15)$, Brazil $(n=13)$, South Africa $(n=10)$, Colombia $(n=9)$, Italy $(n=9)$, Switzerland $(n=4)$, and Belgium, Poland or New Zealand ( $\mathrm{n}=2$ each), and Australia, Austria, Hungary, India, or the Netherlands ( $\mathrm{n}=1$ each). Patients had a median age of 36.0 years, most were women (59\%) and had common variable immunodeficiency disorder (58\%), and approximately half were receiving treatment at home (51\%). Patients for whom caregivers had responded had a median age of 9.0 years, and the majority were male (65\%) and had a variety of diagnoses other than common variable immunodeficiency disorder (68\%). Although similar to patient respondents, approximately half of the patients of respondent caregivers were being treated at home (48\%). Patient demographics are detailed in Table 1.

\section{Current treatment}

The current regular PID treatment for patients was immunoglobulin replacement therapy $(62 \%)$ or immunoglobulin with prophylactic antibiotics $(37 \%)$ or another therapy $(1 \%$; eg, immunoglobulin + cortisone + antivirals). Immunology specialists usually decided on the route of immunoglobulin administration.

Of the 300 respondents, $53 \%$ were either patients or caregivers of patients receiving IVIg therapy and $45 \%$ were patients or caregivers of patients receiving SCIg therapy; $2 \%$ of respondents reported administration by other routes.

Treatment site was linked to route of administration. Most patients taking IVIg had their therapy administered in a hospital (64\% of intravenous respondents) or specialist clinic (11\%), with $15 \%$ receiving treatment at home. Conversely, most patients taking SCIg (94\%) received their therapy at home, with the remainder treated at a regional or local hospital or in a specialist clinic. Time between infusions was also linked with route of administration, with average times between treatments for IVIg respondents being 23 days and 6 days for SCIg respondents. Choice of therapy and therefore route of administration was heavily influenced by the specialist physician. Indeed, the specialist physician was the single main influence in choosing a particular medication for $42 \%$ of respondents, whereas the specialist was reported to be involved and influential to a lesser degree in the choice for $51 \%$ of patients and was not involved in 
Table I Respondent demographics

\begin{tabular}{|c|c|c|c|}
\hline & $\begin{array}{l}\text { All respondents } \\
(n=300)\end{array}$ & Patients $(n=2 \mid 6)$ & $\begin{array}{l}\text { Caregivers answering } \\
\text { on behalf of patients } \\
\text { they care for }(n=84)\end{array}$ \\
\hline \multicolumn{4}{|l|}{ Patient age (years) } \\
\hline $1-10$ & $44(15 \%)$ & $0(0 \%)$ & 44 (52\%) \\
\hline $1 \mathrm{I}-20$ & $50(17 \%)$ & 17 (8\%) & 33 (39\%) \\
\hline $21-30$ & $30(10 \%)$ & $27(13 \%)$ & $3(4 \%)$ \\
\hline $31-50$ & $90(30 \%)$ & 89 (4I\%) & $\mathrm{I}(\mathrm{I \% )}$ \\
\hline $51-60$ & $42(14 \%)$ & 40 (19\%) & $2(2 \%)$ \\
\hline $61+$ & $37(12 \%)$ & $37(17 \%)$ & $0(0 \%)$ \\
\hline Declined to answer & $7(2 \%)$ & $6(3 \%)$ & I (I\%) \\
\hline \multicolumn{4}{|l|}{ Sex } \\
\hline Male/female & 143 (48\%)/I 57 (52\%) & $88(41 \%) / / 28$ (59\%) & $55(65 \%) / 29(35 \%)$ \\
\hline \multicolumn{4}{|l|}{ Diagnosis } \\
\hline CVID & $152(5 \mid \%)$ & $125(58 \%)$ & $27(32 \%)$ \\
\hline Hypogammaglobulinemia & $38(13 \%)$ & $29(13 \%)$ & $9(11 \%)$ \\
\hline X-linked agammaglobulinemia & $36(12 \%)$ & $22(10 \%)$ & $14(17 \%)$ \\
\hline IgG subclass deficiency & $26(9 \%)$ & $14(6 \%)$ & $12(14 \%)$ \\
\hline Hyper IgM syndrome & $9(3 \%)$ & $6(3 \%)$ & $3(4 \%)$ \\
\hline Other & $34(11 \%)$ & $19(9 \%)$ & $15(18 \%)$ \\
\hline Unknown & $5(2 \%)$ & $3(1 \%)$ & $2(2 \%)$ \\
\hline
\end{tabular}

Note: ${ }^{a}$ Demographics shown for caregivers refer to the patient they care for rather than themselves.

Abbreviations: CVID, common variable immunodeficiency; Ig, immunoglobulin.

only $7 \%$ of cases. Patients were the main decision-makers in $13 \%$ of cases (the next most influential group).

Adverse events at the infusion site were experienced by significantly more patients receiving SCIg compared with those receiving IVIg $(P<0.05$; Table 2$)$. Injection site swelling/bumps was very common among patients receiving SCIg (95\% of respondents), although only $7 \%$ of these respondents stated it had a large impact on their life. Other common adverse events for patients receiving SCIg were pain (75\%), itching (54\%), and hardness (55\%) at the infusion site. For most patients, these infusion site reactions had a low or medium impact on their lives and they did not seek to change therapy. However, around one third of this group rated reducing swelling and pain at the infusion site as important aspects to improve, with one quarter mentioning reducing itching.

Treatment-related headaches were experienced by significantly more patients receiving IVIg compared with those receiving SCIg (51\% versus 31\%, $P<0.05$; Table 2 ). Headache was the most common adverse event for patients receiving IVIg, with $11 \%$ of IVIg respondents reporting that it had a large impact on their life. Because of headache, $10 \%$ of IVIg respondents had discussed changing immunoglobulin therapy with their doctor, but only $2 \%$ had actually switched to another therapy. Reducing the incidence of headaches was important for $35 \%$ and $25 \%$ of IVIg respondents and SCIg respondents, respectively, despite a lower proportion of headaches being reported by the latter group.

Table 2 Respondents experiencing immunoglobulin-related injection site reactions and headaches and the impact and importance of reducing these adverse events

\begin{tabular}{|c|c|c|c|c|c|c|}
\hline & \multicolumn{3}{|c|}{ Intravenous respondents $(n=160)$} & \multicolumn{3}{|c|}{ Subcutaneous respondents $(n=134)$} \\
\hline & $\begin{array}{l}\text { Experiencing } \\
\text { AE, n (\%) }\end{array}$ & $\begin{array}{l}\text { Large } \\
\text { impact, } \\
n(\%)\end{array}$ & $\begin{array}{l}\text { Rating reduction } \\
\text { as important, } \\
\text { n (\%) }\end{array}$ & $\begin{array}{l}\text { Experiencing } \\
\text { AE, n (\%) }\end{array}$ & $\begin{array}{l}\text { Large } \\
\text { impact, } \\
\text { n (\%) }\end{array}$ & $\begin{array}{l}\text { Rating reduction } \\
\text { as important, } \\
\mathbf{n}(\%)\end{array}$ \\
\hline Swelling/bumps at infusion site(s) & $45(28)$ & $6(4)$ & $28(18)$ & $125(95)^{\mathrm{a}}$ & $9(7)$ & $42(3 I)$ \\
\hline Pain at infusion site(s) & $70(44)$ & $8(5)$ & $37(23)$ & $100(75)^{\mathrm{a}}$ & $8(6)$ & $47(35)$ \\
\hline Itching at infusion site(s) & $34(21)$ & $3(2)$ & $25(16)$ & $73(54)^{\mathrm{a}}$ & $13(10)$ & $31(23)$ \\
\hline Hardness at infusion site(s) & $37(23)$ & $8(5)$ & - & $74(55)^{\mathrm{a}}$ & $10(7)$ & - \\
\hline Headaches & $8 \mathrm{I}(5 \mathrm{I})^{\mathrm{a}}$ & $18(11)$ & $55(34)$ & $4 I(3 I)$ & $10(7)$ & $33(25)$ \\
\hline Other AEs & $72(45)^{\mathrm{a}}$ & $26(16)$ & - & $44(33)$ & $20(15)$ & - \\
\hline
\end{tabular}

Note: ${ }^{\mathrm{P}}<0.05$ for intravenous versus subcutaneous group.

Abbreviation: $\mathrm{AE}$, adverse event. 
Reasons for stopping IVIg or SCIg treatment were examined in the subgroup of patients who had switched routes of administration. IVIg respondents who had previously tried SCIg therapy $(n=31)$ were most likely to have stopped because of adverse events $(61 \%)$ and wanting a longer time between doses $(32 \%)$. SCIg respondents who had tried IVIg therapy $(\mathrm{n}=104)$ most commonly stopped because of the inconvenience of traveling to the infusion center (51\%), wanting to be treated at home (43\%), or because their doctor recommended they change treatment (48\%).

Most patients (76\%) were satisfied with their current therapy; however, significant differences in satisfaction were seen when comparing SCIg with IVIg administration. Overall, SCIg respondents were more satisfied with treatment than IVIg respondents ( $83 \%$ versus $69 \%$, respectively; $P<0.05)$. Compared with patients receiving IVIg, those receiving SCIg liked the ability to self-administer $(91 \%$ versus $31 \%$ ), being able to fit treatment into their schedule ( $90 \%$ versus $58 \%$ ), and the reduced amount of time taken to administer treatment (65\% versus $39 \%$ ). Waiting time at the hospital or clinic was the factor with which IVIg respondents were most commonly dissatisfied (14\%), while number of needles per month (19\%) and frequency of infusions (12\%) caused most dissatisfaction for SCIg respondents. The overall convenience of treatment was "liked" by significantly more SCIg respondents than IVIg respondents ( $88 \%$ versus $60 \%$, respectively; $P<0.05$ ).

\section{Living with PID}

Using the EuroQoL 5 Dimensions questionnaire, patients, their caregivers, or both indicated the health of the patient "today" with regard to mobility, self-care, ability to perform usual activities, pain, and anxiety/depression. Respondents reported a mean score of 71.6 (on a scale from 0 [worst health state] to 100 [best health state]). Results were similar by country and by mode of immunoglobulin administration (Figure 1).

In the absence of available European norms, average scores were below US population norms across the SF-12 physical and mental components for patients with PID (Figure 2). ${ }^{18}$ Compared with US norms scaled to a mean of 50, SF-12 summary scores for PID were 40.7 for the physical component and 46.0 for the mental component. Scores for the individual components ranged from 37.9 (general health) to 46.5 (mental health). No statistically significant difference was seen between those receiving intravenous and subcutaneous infusions.

Immunoglobulin treatment enabled patients to enjoy a relatively high degree of normality. Sixty-six percent of IVIg respondents and $69 \%$ of SCIg respondents reported missing 10 or fewer work/school days due to ill health during the previous 6 months; $35 \%$ of IVIg respondents and 37\% of SCIg respondents reported no absences. No significant difference was seen in the number of unscheduled doctor visits based on type of administration (IVIg versus SCIg).

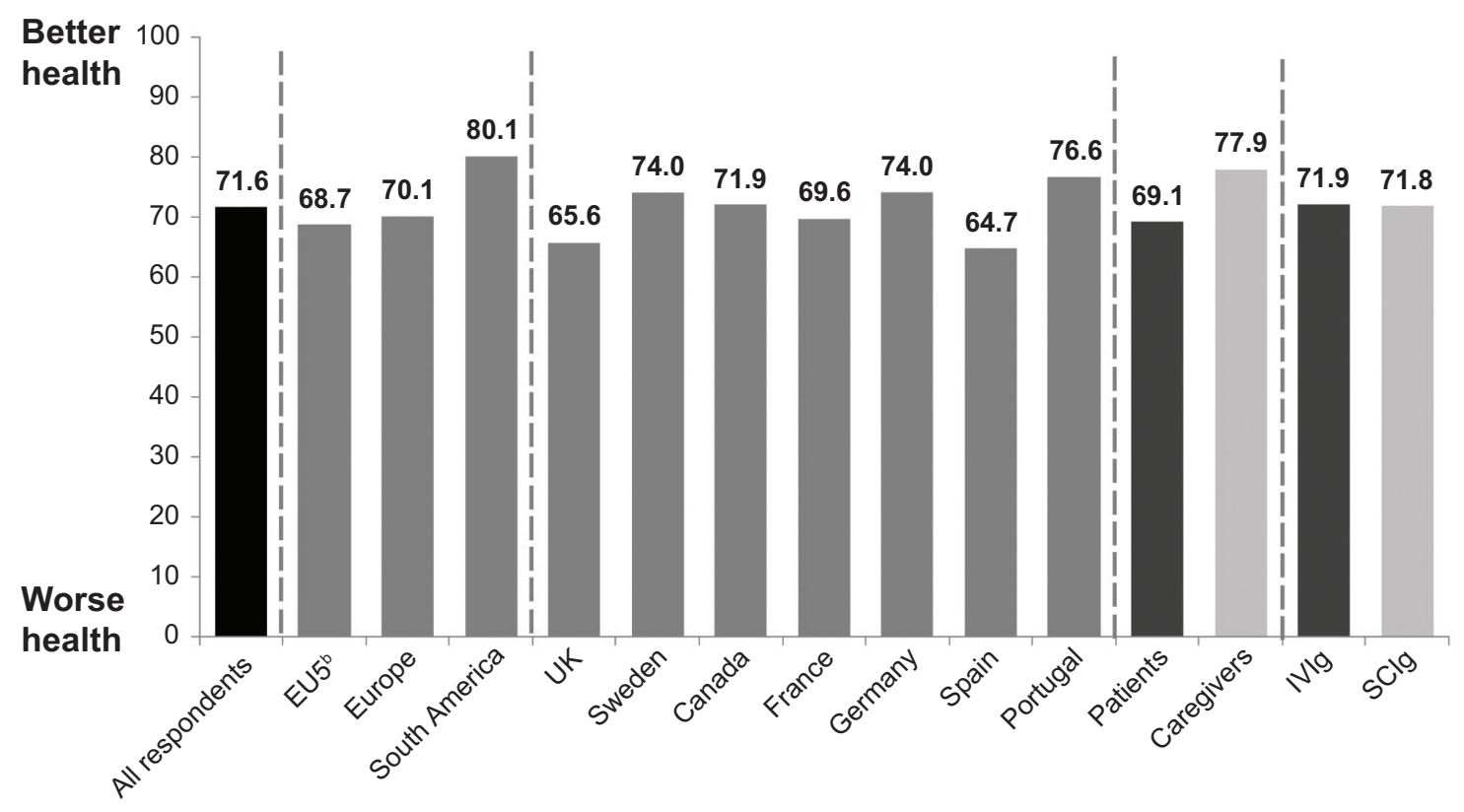

Figure I Health of patients with primary immunodeficiency disease by country and route of immunoglobulin administration (visual analog scale). ${ }^{a}$

Notes: ${ }^{a} E Q-5 D$ questionnaire; 'EU5 indicates UK, France, Germany, Spain, and Italy.

Abbreviations: Ig, immunoglobulin; IV, intravenous; SC, subcutaneous; EQ-5D, EuroQoL 5 Dimensions. 


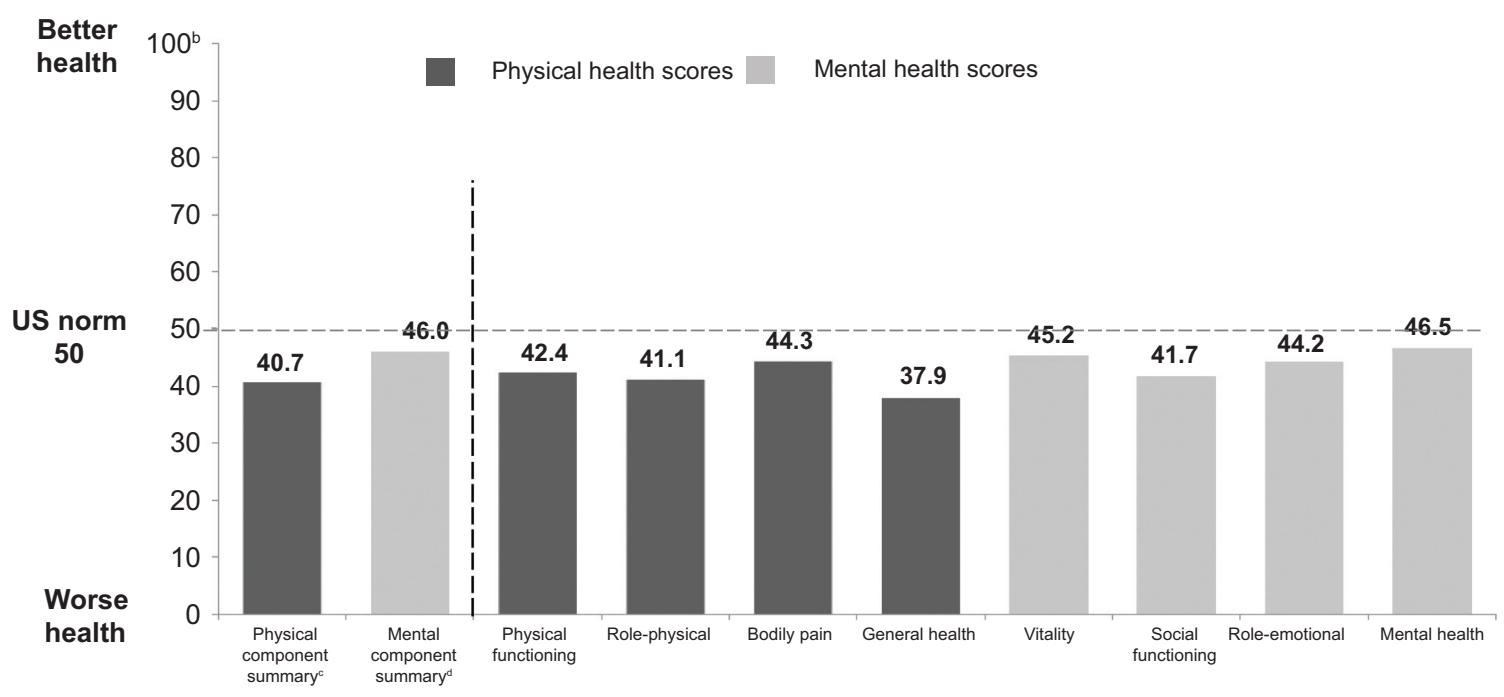

Figure 2 I2-Item Short Form Health Survey physical and mental norm-based ${ }^{\mathrm{a}}$ summary and component scores for patients with primary immunodeficiency diseases treated with immunoglobulin.

Notes: ${ }^{U}$ sing norm-based scoring, each scale is scored to have the same mean (50) and standard deviation (I0) as the general US population; ${ }^{b}$ original scale is 0-100, with 100 being better health; 'includes several elements (ie, limitations in self care, physical, social, and role activities; physical well-being; and rating of health); ${ }^{\text {includes several }}$ elements (ie, psychological distress, social and role disability due to emotional problems and general health rating).

\section{Treatment preferences (conjoint analysis)}

Preference weights for treatment attributes decided by patients and caregivers are shown in Figure 3. Both patients and caregivers significantly preferred selfadministration to administration by a health care professional $(P<0.05)$ and administration at home rather than in a doctor's office, clinic, or hospital $(P<0.05)$. There was a strong preference on the part of all respondents for monthly administration when compared with either fortnightly or weekly administration $(P<0.05)$. Preferences for number of needle sticks per treatment (one, two, four) were significantly different from each other $(P<0.05)$, and as expected, both patients and caregivers preferred fewer needle sticks. Nevertheless, a significantly stronger preference for one needle stick was also recorded for caregivers more than for patients $(P<0.05)$. Preferences for each treatment duration level per administration (2, 4, and 6 hours) were also significantly different from each other, and also as expected, both groups preferred a shorter duration $(P<0.05)$.

A further analysis was undertaken of the combined patient and caregiver responses depending on their route

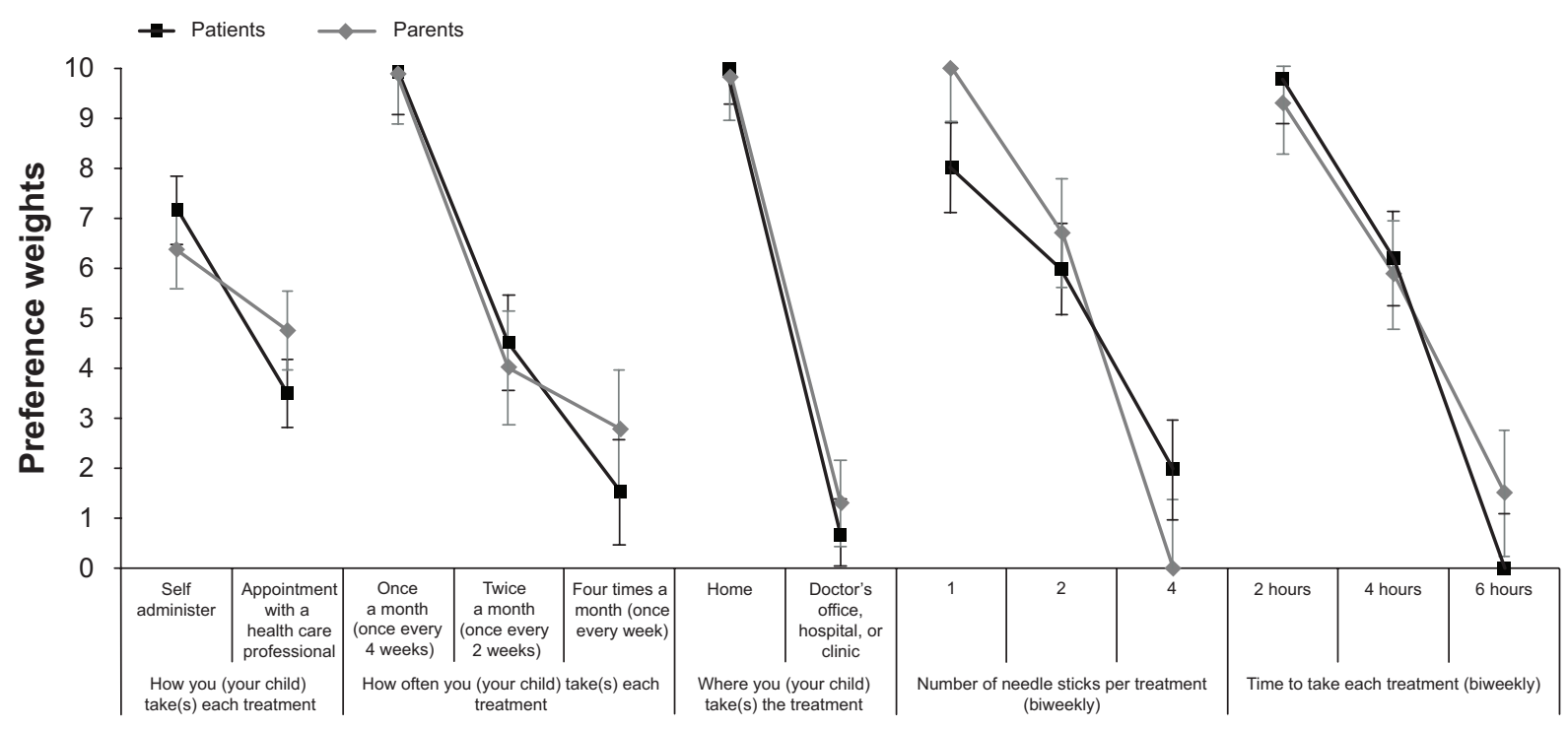

Figure 3 Preference weights for treatment attributes (point estimates and $95 \%$ confidence intervals) decided by patients $(n=216)$ and caregivers ( $n=84$ ). 
of administration, ie, IVIg or SCIg. Patients receiving SCIg as well as their caregivers significantly preferred selfadministration to administration by a health care professional $(P<0.05)$, but this was not the case for users of IVIg and their caregivers $(P>0.05)$. In contrast, both administration cohorts significantly preferred treatment in the home compared with treatment in the doctor's office, clinic, or hospital $(P<0.05)$.

Significant differences were noted for the entire sample toward preferences for monthly treatment $(P<0.05)$ and the shortest duration of treatment $(P<0.05)$. Similarly, all respondents showed a preference for treatment with one needle stick $(P<0.05)$, although the difference between one and two needle sticks for the subcutaneous cohort did not achieve statistical significance $(P>0.05)$. Significant differences were also seen between the IVIg and SCIg groups in regard to other attributes. For example, IVIg respondents had a stronger preference for once-a-month treatment and a single needle stick per treatment (both $P<0.05$ ), while SCIg respondents had a stronger preference for self-administration and treatment at home (both $P<0.05$ ).

\section{Discussion}

Treatment of the most frequently encountered PIDs is primarily based on immunoglobulin replacement therapy and protection from infection. ${ }^{4}$ There is good evidence that immunoglobulin replacement therapy prolongs survival and reduces morbidity, and that administration by either the intravenous or subcutaneous route contributes to an improvement in HRQoL. ${ }^{9}$ While immunoglobulin treatment has significant benefits for patients with PID, the results of our survey indicate that the condition still impacts on HRQoL. Furthermore, while current immunoglobulin treatments were generally associated with high levels of satisfaction, significant differences were seen with regard to patient satisfaction and acceptability of certain convenience aspects of immunoglobulin treatment between patients treated with IVIg and those treated with SCIg. SCIg therapy achieved greater patient satisfaction, which may be attributed to better therapy convenience and greater independence associated with this administration route. ${ }^{19}$ Specialist physicians in our survey clearly had a dominant role in the choice of therapy and therefore have a role in improving patient-tailored treatments. Furthermore, our findings indicate that there is still room for improvement in immunoglobulin therapy regardless of administration route. The subgroup of patients who switched routes of administration (from SCIg to IVIg or from IVIg to SCIg) indicated that unfavorable features causing them to switch from SCIg to IVIg were adverse events and frequency of dosing, while those stopping IVIg therapy mentioned inconvenience of traveling to the infusion center and a desire to be treated at home. A recently released IPOPI position statement highlights the importance of ensuring that patients with PID have access to the most appropriate immunoglobulin therapy according to their disease and personal conditions. ${ }^{20}$

Consistent with the findings of this survey, a systematic review and meta-analysis of 47 studies comparing the efficacy and safety of IVIg and SCIg showed a significant preference for SCIg over IVIg in terms of adverse events (odds ratio 0.09 , range $0.07-0.11 ; P<0.001$ ). ${ }^{21}$ Indeed, SCIg therapy is thought to be better tolerated than IVIg with regard to systemic reactions, ${ }^{22}$ although some studies report no significant differences in adverse event rates between SCIg and IVIg replacement therapy. ${ }^{23,24}$ Our study findings may reflect the imbalance of subcutaneous to intravenous and intravenous to subcutaneous switches; only $19 \%$ of patients currently receiving IVIg therapy had previously tried SCIg therapy, while most patients currently on SCIg therapy (78\%) had previously tried IVIg therapy. For most of the patients in our survey, adverse events had only low or medium impact on their life and they neither discussed them with their health care provider nor sought to change therapy. However, treatment-related infusion site reactions were reported, particularly by patients receiving SCIg therapy, while those on IVIg therapy had more headaches. These findings were not unexpected because such events are frequently reported when immunoglobulin is administered by these routes. ${ }^{8,25}$ Of note, $19 \%$ of patients with PID are reported to discontinue immunoglobulin therapy because of treatment-related adverse events. ${ }^{26}$

Our conjoint analysis revealed that patients and caregivers preferred self-administration to administration by a health care professional, monthly over fortnightly or weekly administration, treatment in the home rather than in a health care organization, a shorter duration of administration, and fewer needle sticks. Similar results were identified when comparing respondents according to whether they received IVIg or SCIg; however, respondents receiving subcutaneous therapy had a relatively higher preference for self-administration. The flexibility of home therapy contributes to improved HRQoL and gives a sense of independence to patients and families. ${ }^{8}$ However, our study did not examine the effect of home versus hospital administration of therapy on HRQoL.

The results of our analysis were corroborated by a similar survey of immunoglobulin administration preferences conducted in 218 patient and caregivers in the USA. ${ }^{10}$ The USA 
study also demonstrated that both patients and caregivers preferred administration in the home setting, a monthly frequency, fewer needle sticks, and a shorter treatment duration. Although the mode of administration was rated as the least important attribute, caregivers showed strongest preference for self-administration, whereas patients showed similar preferences between the two administration modes.

A number of limitations should be considered when interpreting these survey data. Patients and caregivers who completed the survey were recruited through NMOs affiliated to the IPOPI and might not represent all people with PID treated with immunoglobulin therapy. The study was also limited to 300 respondents from 21 countries, so its findings may not be representative for any one specific country. The large differences in number of responses between countries depended on NMO membership, cultural differences, and the individual's willingness to take part in the study. Although some IPOPI NMOs are staffed, many of these patient organizations are run by volunteers and have few resources to dedicate to such surveys, hence the differences in level of participation. Moreover, because of the large number of countries and relatively small number of respondents per country, the health scores for PID participants were compared with a single measurement rather than with their own population. In addition, recall failure, misunderstanding of the question, or both, can contribute to data inaccuracies. However, our survey reflects the current difficulties of everyday living and inconvenience of treatment administration faced by patients with PID.

Calls for individualization of patient treatment in PID highlight the importance of patient input into decisionmaking when choosing the most appropriate therapy. ${ }^{27,28}$ In this respect, patient preference and patient-reported outcome studies in this and other therapeutic areas have underscored the importance of administration as a key attribute of treatment preferences rating even above clinical outcomes. ${ }^{29,30}$ For PID in particular, a high level of clinical consensus exists that optimal management requires a multifactorial, individualized approach to complement evidence-based guidelines and policies. ${ }^{31}$ Our study supports this approach, since it shows that treatment preferences indeed vary by patient and caregiver, so ideal treatment needs to be individualized.

\section{Conclusion}

This multinational study shows that patients with PID have high levels of satisfaction with current immunoglobulin replacement therapy, whether by intravenous or subcutaneous administration, reflecting the benefits of treatment to their health and well-being. It highlights the importance of providing access to different treatment options and modes of administration to ensure individual patient needs are best met. Nevertheless, there remains room for improvement with therapy for patients still below the physical and mental HRQoL population norms. In this survey sample, the desire for monthly frequency, ability to administer at home, selfadministration, shorter administration duration, and fewer needle sticks highlights opportunities to improve treatment and satisfy unmet patient needs. There is an opportunity to develop new treatments that may provide additional options to address patient needs and improve the HRQoL of those living with PID, bearing in mind the need to take into account individual patient requirements, preferences, and tolerability patterns. Replication of our study findings in larger patient cohorts would underscore this conclusion.

\section{Acknowledgments}

We would like to acknowledge the employees of Bryter, an independent market research company that carried out this survey, analyzed the data, and prepared the report for the IPOPI. We would also like to thank Sue Libretto, David Peters, and Gauri Saal of Rx Communications for editorial assistance.

\section{Disclosure}

This study was funded by Baxter Healthcare SA.

\section{References}

1. Al-Herz W, Bousfiha A, Casanova JL, et al. Primary immunodeficiency diseases: an update on the classification from the International Union of Immunological Societies Expert Committee for Primary Immunodeficiency. Front Immun. 2011;2:54.

2. McCusker C, Warrington R. Primary immunodeficiency. Allergy Asthma Clin Immunol. 2011;7 Suppl 1:S11.

3. Notarangelo LD. Primary immunodeficiencies. J Allergy Clin Immunol. 2010;125(2 Suppl 2):S182-S194.

4. Cunningham-Rundles C. Key aspects for successful immunoglobulin therapy of primary immunodeficiencies. Clin Exp Immunol. 2011; 164 Suppl 2:16-19.

5. Misbah S, Sturzenegger MH, Borte M, et al. Subcutaneous immunoglobulin: opportunities and outlook. Clin Exp Immunol. 2009; 158 Suppl 1:51-59.

6. Berger M. Subcutaneous immunoglobulin replacement in primary immunodeficiencies. Clin Immunol. 2004;112:1-7.

7. Gardulf A. Immunoglobulin treatment for primary antibody deficiencies: advantages of the subcutaneous route. Bio Drugs. 2007;21:105-116.

8. Skoda-Smith S, Torgerson TR, Ochs HD. Subcutaneous immunoglobulin replacement therapy in the treatment of patients with primary immunodeficiency disease. Ther Clin Risk Manag. 2010;6:1-10.

9. Wood P. Human normal immunoglobulin in the treatment of primary immunodeficiency diseases. Ther Clin Risk Manag. 2012;8:157-167.

10. Mohamed AF, Kilambi V, Luo MP, Iyer RG, Li-McLeod JM. Patient and parent preferences for immunoglobulin treatments: a conjoint analysis. J Med Econ. 2012;15:1-9.

11. QualityMetric. SF-12v2 health survey. Available from: http://www.qualitymetric.com/WhatWeDo/SFHealthSurveys/SF12v2HealthSurvey/ tabid/186/Default.aspx. Accessed May 7, 2013. 
12. QualityMetric. SF-10 Health Survey for Children. Available from: http://www.qualitymetric.com/WhatWeDo/PediatricHealthSurveys/ SF10HealthSurveyforChildren/tabid/195/Default.aspx. Accessed February 28, 2014.

13. EuroQol Group. About EQ-5D. Available from: http://www.euroqol. org/about-eq-5d.html. Accessed December 23, 2013.

14. McFadden D, Train K. Mixed MNL models of discrete response. J Appl Econ. 2000;15:447-470.

15. Hauber AB, Mohamed AF, Johnson FR, Meddis D, Wagner S, O'Dowd L. Quantifying asthma patient preferences for onset of effect of combination inhaled corticosteroids and long-acting beta2agonist maintenance medications. Allergy Asthma Proc. 2009;30: $139-147$.

16. Hauber AB, Mohamed AF, Watson ME, Johnson FR, Hernandez JE. Benefits, risks and uncertainty: references of antiretroviral-naive African Americans for HIV treatment. AIDS Patient Care STDS. 2009; 23:29-34.

17. Mohamed AF, Hauber AB, Neary MP. Patient benefit-risk preferences for targeted agents in the treatment of renal cell carcinoma. Pharmacoeconomics. 2011;29:977-988.

18. Ware JE, Kosinski M, Turner-Bowker DM, Gandek B. How to Score Version 2 of the SF-12 Health Survey. Lincoln, RI, USA: QualityMetric Inc; 2002.

19. Gardulf A, Nicolay U, Math D, et al. Children and adults with primary antibody deficiencies gain quality of life by subcutaneous IgG selfinfusions at home. J Allergy Clin Immunol. 2004;114:936-942.

20. International Patient Organisation for Primary Immunodeficiencies. Position statement: access to immunoglobulin therapies for patients living with a primary immunodeficiency; 2012. Available from: http:// www.ipopi.org/index.php?mact=News,cntnt01, detail,0\&cntnt01 articl eid $=218 \&$ cntnt 01 origid $=38 \&$ cntnt 01 detailtemplate $=$ detalj $\&$ cntnt 01 ret urnid=63. Accessed May 7, 2013.

21. Abolhassani H, Sadaghiani MS, Aghamohammadi A, Ochs HD, Rezaei N. Home-based subcutaneous immunoglobulin versus hospitalbased intravenous immunoglobulin in treatment of primary antibody deficiencies: systematic review and meta analysis. J Clin Immunol. 2012;32:1180-1192.
22. Gardulf A, Andersen V, Björkander J, et al. Subcutaneous immunoglobulin replacement in patients with primary antibody deficiencies: safety and costs. Lancet. 1995;345:365-369.

23. Chapel HM, Spickett GP, Ericson D, Engl W, Eibl MM, Bjorkander J. The comparison of the efficacy and safety of intravenous versus subcutaneous immunoglobulin replacement therapy. J Clin Immunol. 2000;20:94-100.

24. García JM, Español T, Gurbindo MD, Casas CC. Update on the treatment of primary immunodeficiencies. Allergol Immunopathol (Madr). 2007; 35:184-192.

25. Bonilla FA. Intravenous immunoglobulin: adverse reactions and management. J Allergy Clin Immunol. 2008;122:1238-1239.

26. Immune Deficiency Foundation. Primary immunodeficiency diseases in America: 2007. The third national survey of patients; 2009. Available from: http://primaryimmune.org/about-primary-immunodeficiencydiseases/idf-publications?aid-1264\&pid=275\&sa=1. Accessed May 7, 2013.

27. Bonilla FA. IgG replacement therapy, no size fits all. J Clin Immunol. 2011;139:107-109.

28. Orange JS. Clinical update in immunoglobulin therapy for primary immunodeficiency diseases. Clinical Focus on Primary Immunodeficiencies. 2011;14:1-9. Available from: http://primaryimmune.org/ wp-content/uploads/2011/04/Clinical-Update-in-ImmunoglobulinTherapy-for-Primary-Immunodeficiency-Diseases.pdf. Accessed December 23, 2013.

29. Crawford B, Dalal AA, Stanford R, Bayliss MS, Lim J, Philpot E. Validation of the Experience with Allergic Rhinitis Nasal Spray Questionnaire (EARNS_Q): a patient reported outcomes (PRO) questionnaire to measure experience and preference with nasal sprays. J Allergy Clin Immunol. 2008;121(2 Suppl):S106.

30. Fasth A, Nyström J. Quality of life and health-care resource utilization among children with primary immunodeficiency receiving home treatment with subcutaneous human immunoglobulin. J Clin Immunol. 2008;28:370-378

31. Navarro RP, Ballow M, Fenrick B, Pezalla EJ. Considerations for the optimal use of immunoglobulin. Am J Manag Care. 2012;18 (4 Suppl):S67-S78.
Patient Preference and Adherence

\section{Publish your work in this journal}

Patient Preference and Adherence is an international, peer-reviewed, open access journal that focusing on the growing importance of patient preference and adherence throughout the therapeutic continuum. Patient satisfaction, acceptability, quality of life, compliance, persistence and their role in developing new therapeutic modalities and compounds to optimize

\section{Dovepress}

clinical outcomes for existing disease states are major areas of interest for the journal. This journal has been accepted for indexing on PubMed Central. The manuscript management system is completely online and includes a very quick and fair peer-review system, which is all easy to use. Visit http://www. dovepress.com/testimonials.php to read real quotes from published authors. 